\title{
Dinâmica de Decomposição e Nutrientes em Plantio de Eucalyptus urophylla $\times$ Eucalyptus globulus no Sul do Brasil
}

\author{
Márcio Viera ${ }^{1}$, Mauro Valdir Schumacher ${ }^{2}$, Marcos Vinicius Winckler Caldeira $^{3}$ \\ ${ }^{1}$ Departamento Multidisciplinar, Universidade Federal de Santa Maria - UFSM, Silveira Martins/RS, Brasil \\ ${ }^{2}$ Departamento de Ciências Florestais, Universidade Federal de Santa Maria - UFSM, Santa Maria/RS, Brasil \\ ${ }^{3}$ Departamento de Ciências Florestais e da Madeira, Universidade Federal do Espírito Santo - UFES, \\ Jerônimo Monteiro/ES, Brasil
}

\section{RESUMO}

Objetivou-se, neste trabalho, determinar o estoque de serapilheira acumulada, sua decomposição e a taxa de disponibilização de nutrientes em um plantio híbrido de Eucalyptus urophylla $\times$ Eucalyptus globulus, em Eldorado do Sul-RS. Foram demarcadas quatro parcelas $(20 \mathrm{~m} \times 20 \mathrm{~m})$ e, em cada uma delas, foram coletadas estacionalmente (entre março de 2007 e dezembro de 2010) quatro amostras de forma aleatória. A quantidade de serapilheira estocada sobre o solo foi, em média, de $14,0 \mathrm{Mg} \mathrm{ha}^{-1}$. O coeficiente de decomposição foi de 0,54, com tempo médio para a renovação $(1 / \mathrm{K})$ de 1,86 anos e tempo médio para decomposição de 50 e $95 \%$ da serapilheira em 1,29 e 5,59 anos, respectivamente. O potássio apresentou o maior coeficiente de devolução (0,52), seguido por $\mathrm{B}>\mathrm{Mg}>\mathrm{S}=\mathrm{Mn}>\mathrm{P}>\mathrm{N}=\mathrm{Ca}>\mathrm{Zn}>\mathrm{Cu}$; consequentemente, o potássio apresentou menor tempo médio de residência na serapilheira $(0,91$ anos).

Palavras-chave: ciclagem de nutrientes, coeficiente de decomposição, disponibilização de nutrientes.

\section{Litter Decomposition and Nutrients in Eucalyptus urophylla $\times$ Eucalyptus globulus Hybrid Stand in Southern Brazil}

\begin{abstract}
In this study, we aimed to determine the litter accumulation stock, its decomposition and nutrient release in an Eucalyptus urophylla $\times$ Eucalyptus globulus hybrid plantation in the municipality of Eldorado do Sul, Rio Grande do Sul state. Four plots were demarcated $(20 \mathrm{~m} \times 20 \mathrm{~m})$ and four samples were seasonally sampled (from March 2007 to December 2010) at random. Average litter accumulation on soil was $14.0 \mathrm{Mg} \mathrm{ha}^{-1}$. Litter accumulated showed 0.54 decomposition coefficient with average renewal time $(1 / \mathrm{K})$ of 1.86 years and average time to litter decomposition of 50 , and $95 \%$ of litter in 1.29 and 5.59 years, respectively. Potassium presented the highest turnover (0.52), followed by B $>\mathrm{Mg}>\mathrm{S}=\mathrm{Mn}>\mathrm{P}>\mathrm{N}=\mathrm{Ca}>\mathrm{Zn}>\mathrm{Cu}$ and consequently the lowest average residence time in the litter ( 0.91 years).
\end{abstract}

Keywords: nutrient cycling, decomposition coefficient, nutrient release. 


\section{INTRODUÇÃO}

A serapilheira acumulada na superfície do solo constitui-se de uma camada de detritos vegetais (folhas, ramos, caules, cascas, frutos e flores) e animais. Ela contribui, juntamente com os demais compartimentos florestais, para a interceptação da água da chuva e o seu armazenamento no solo, e para o aumento das taxas de infiltração e condicionamento dos fluxos superficiais (Olson, 1963). A quantidade de serapilheira acumulada e o processo de decomposição estão relacionados com fatores abióticos e bióticos, tais como: tipo de vegetação; altitude; latitude; precipitação; temperatura; regimes de luminosidade; relevo; deciduidade; estágio sucessional; disponibilidade hídrica, e características do solo (Vitousek \& Sanford, 1986; Figueiredo Filho et al., 2003; Viera \& Schumacher, 2010). A concentração e a quantidade de nutrientes na serapilheira variam em função dos seguintes aspectos: tipo de solo; vegetação; densidade populacional; habilidade da espécie em absorver, utilizar e translocar os nutrientes antes da senescência foliar; proporção de folhas em relação aos demais componentes; habitat natural (condições edafoclimáticas), e idade das árvores (Pritchett, 1979; Koehler et al., 1987; Neves et al. 2001; Viera \& Schumacher, 2009).

No processo de decomposição da serapilheira, a liberação de nutrientes depende da qualidade da serapilheira, de variáveis macro e microclimáticas, e da atividade biótica. Os fatores climáticos que mais influenciam na decomposição da serapilheira são a temperatura e a umidade do solo (Dutta \& Agrawal, 2001; Guo \& Sims, 2002; Campos et al., 2004). Além disso, outro fator primordial responsável pela maior ou menor taxa de decomposição é a composição estrutural dos tecidos, pois tecidos com maiores percentuais de celulose, hemicelulose e lignina são mais resistentes à decomposição do que tecidos mais pobres nesses materiais (Haag, 1985; O'Connell, 1988).

A decomposição da serapilheira pode ser dividida em três fases simultâneas: lixiviação, intemperismo e ação biológica (Mason, 1980). Segundo o autor, a lixiviação inclui a perda rápida de material solúvel do detrito pela ação da água da chuva ou do fluxo de água. O intemperismo, salienta o autor, é a ruptura mecânica dos detritos devido a fatores físicos, como abrasão pelo vento. Por fim, ele menciona que a ação biológica resulta na fragmentação gradual e na oxidação dos detritos por organismos vivos. Como resultado dessas fases, ocorre a circulação de nutrientes no sistema solo-planta (ciclagem biogeoquímica), tendo a serapilheira como principal meio de transferência (Viera et al., 2010) e, consequentemente, de disponibilização de nutrientes para reabsorção pelas plantas (Viera \& Schumacher, 2010).

A quantidade de serapilheira acumulada sobre o solo florestal em plantios de eucalipto não varia durante o ano, mas apenas entre as espécies ou os materiais genéticos utilizados (Zaia \& GamaRodrigues, 2004) e a idade do povoamento (Witschoreck \& Schumacher, 2000). Em estudo realizado por Zaia \& Gama-Rodrigues (2004), as taxas de decomposição para diferentes espécies de eucalipto (Eucalyptus grandis, E. camaldulensis e E. pellita) variou entre 0,51 e 1,0, valores considerados baixos quando comparados com florestas tropicais, nas quais os valores de $\mathrm{K}$ geralmente são superiores a 1,0 (Pires et al., 2006). A taxa de decomposição da serapilheira está diretamente relacionada com o processo de disponibilização de nutrientes. Em razão disso, o presente estudo objetivou determinar o estoque de serapilheira acumulada, sua decomposição e a taxa de disponibilização de nutrientes em um plantio de Eucalyptus urophylla $\times$ Eucalyptus globulus, em Eldorado do Sul-RS.

\section{MATERIAL E MÉTODOS}

\subsection{Caracterização da área de estudo}

A área experimental foi instalada em um plantio de Eucalyptus urophylla $\times$ Eucalyptus globulus, localizado no Horto Florestal Terra Dura no município de Eldorado do Sul-RS, em área pertencente à Empresa Celulose Riograndense (CMPC). Sua localização geográfica é $30^{\circ} 10^{\prime} 31,21^{\prime \prime} \mathrm{S}$ e $51^{\circ} 36^{\prime} 17,85^{\prime \prime} \mathrm{O}$.

Segundo a classificação climática de Köppen, o tipo de clima predominante é o Cfa (subtropical úmido), com verão quente e precipitação em todos os 
meses do ano (Moreno, 1961). A temperatura média anual é de $20{ }^{\circ} \mathrm{C}$, com precipitação pluviométrica anual de $1.500 \mathrm{~mm}$ (FEPAGRO, 2012). As variações mensais de precipitação e de temperatura para os anos de 2007 até 2010, na região de estudo, encontram-se na Figura 1, que mostra o diagrama climático do período de estudo, no município de Eldorado do Sul-RS.
O solo, na área experimental, foi classificado como Argissolo Vermelho Distrófico típico (Streck et al., 2008), cujos atributos químicos, em diferentes profundidades, estão apresentados na Tabela 1. A amostragem do solo, para análise química e densidade, foi realizada por meio da abertura de nove trincheiras de $1,0 \mathrm{~m} \times 1,0 \mathrm{~m} \times 1,3 \mathrm{~m}$. As análises dos nutrientes disponíveis no solo foram

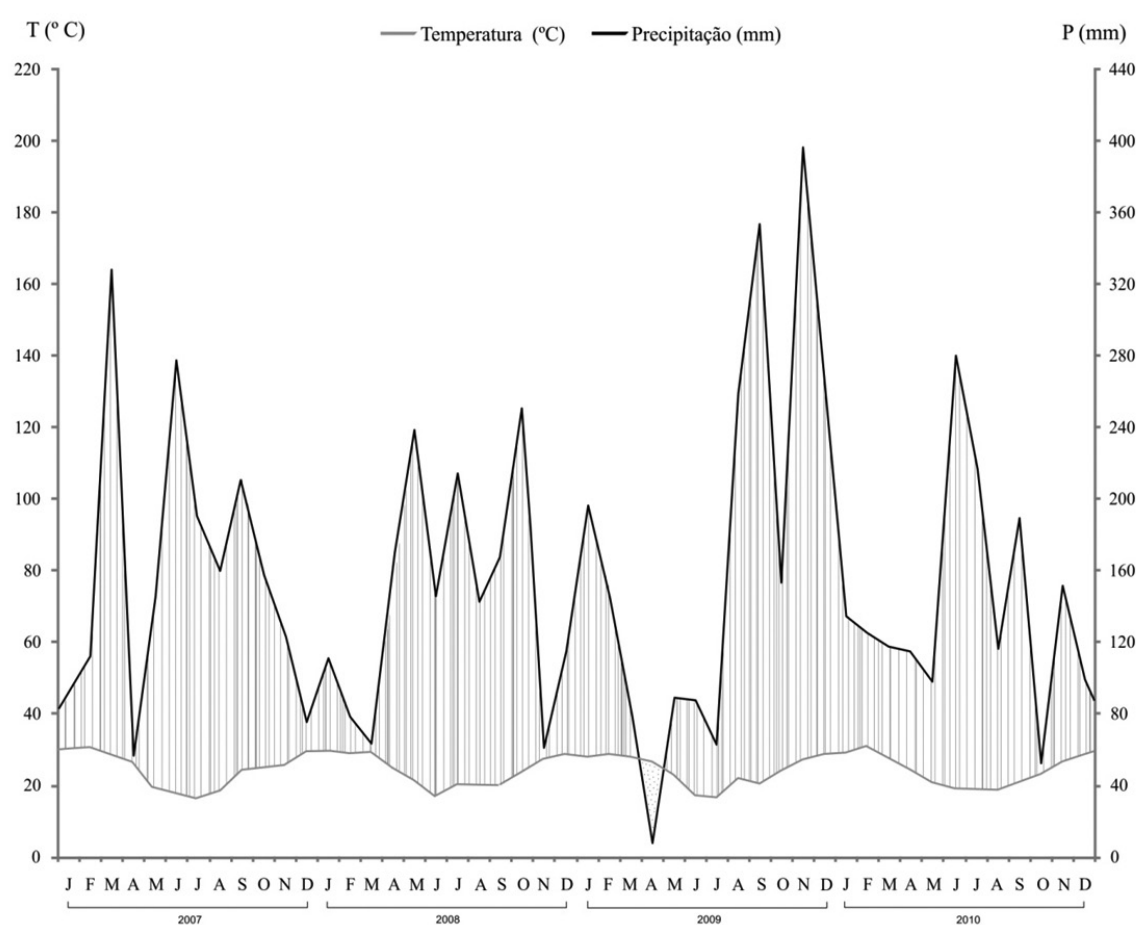

Figura 1. Diagrama climático da região de Eldorado do Sul durante o período de estudo (2007 a 2010). Fonte: Estação Experimental Agronômica do Departamento de Plantas Forrageiras e Agrometeorologia da UFRGS, em Eldorado do Sul-RS.

Figure 1. Climatic diagram of Eldorado do Sul region during the study (2007 to 2010). Source: Estação Experimental Agronômica do Departamento de Plantas Forrageiras e Agrometeorologia da UFRGS, em Eldorado do Sul-RS, Brazil.

Tabela 1. Atributos químicos do solo na área experimental localizada no município de Eldorado do Sul-RS.

Table 1. Soil chemical attributes in the experimental area located in Eldorado do Sul-RS.

\begin{tabular}{|c|c|c|c|c|c|c|c|}
\hline \multirow{2}{*}{ Prof. (cm) } & MO & \multirow{2}{*}{$\mathrm{pH}\left(\mathrm{H}_{2} \mathrm{O}\right)$} & \multirow{2}{*}{$\mathrm{pH}\left(\mathrm{CaCl}_{2}\right)$} & $\mathbf{P}$ & K & m & V \\
\hline & $(\%)$ & & & \multicolumn{2}{|c|}{$\mathrm{mg} \mathrm{dm}^{-3}$} & \multicolumn{2}{|c|}{$(\%)$} \\
\hline $0-25$ & 2,46 & 4,64 & 3,89 & 1,86 & 73,46 & 79,32 & 20,68 \\
\hline $25-50$ & 1,94 & 4,73 & 3,93 & 0,85 & 71,92 & 78,44 & 21,56 \\
\hline $50-75$ & 0,90 & 4,81 & 3,97 & 0,83 & 55,27 & 76,22 & 23,78 \\
\hline $75-100$ & 0,48 & 4,87 & 4,01 & 0,75 & 37,80 & 81,47 & 18,53 \\
\hline \multirow{2}{*}{ Prof. $(\mathrm{cm})$} & $\mathrm{CO}$ & $\mathbf{N}$ & Al & CTC $_{\text {eft }}$ & $\mathrm{Ca}$ & Mg & $\mathbf{N a}$ \\
\hline & \multicolumn{2}{|c|}{$\%$} & \multicolumn{5}{|c|}{$\mathrm{cmol}_{\mathrm{cm}} \mathrm{dm}^{-3}$} \\
\hline $0-25$ & 1,23 & 0,08 & 4,13 & 7,47 & 0,34 & 0,41 & 0,28 \\
\hline $25-50$ & 0,97 & 0,07 & 5,38 & 6,09 & 0,25 & 0,42 & 0,25 \\
\hline $50-75$ & 0,45 & 0,03 & 4,83 & 4,72 & 0,24 & 0,38 & 0,22 \\
\hline $75-100$ & 0,24 & 0,02 & 4,45 & 8,02 & 0,24 & 0,30 & 0,30 \\
\hline
\end{tabular}


determinadas por meio do ICP (Inductively Coupled Plasma) e do N total, pelo CNS (Analysator Carlo Erba NA 1500).

Segundo a SBCS (2004), o teor de matéria orgânica no solo é baixo ( $\leq 2,5 \%)$; o pH e o P são muito baixos ( $\leq 5,0 \mathrm{e} \leq 3,0 \mathrm{mg} \mathrm{dm}^{-3}$, respectivamente); o $\mathrm{K}$ é de médio a alto $\left(40-120 \mathrm{mg} \mathrm{dm}^{-3}\right)$; o Ca e o $\mathrm{Mg}$ são baixos ( $\leq 2,0 \mathrm{e} \leq 0,5 \mathrm{cmol}_{\mathrm{c}} \mathrm{dm}^{-3}$, respectivamente); a saturação por Alé alta ( $>20 \%$ ) e a saturação por bases é muito baixa $(<45 \%)$. Com isso, de maneira geral, o solo da área experimental é de baixa fertilidade.

O solo é caracterizado por textura média/argilosa (Tabela 2) com cascalhos ou argilosa com cascalhos, em relevo de suave ondulado a ondulado.

A implantação do povoamento foi realizada por meio de preparo reduzido do solo na linha de plantio, com utilização de um subsolador, até uma profundidade média de $40 \mathrm{~cm}$, durante o mês de julho de 2001. Foram utilizadas mudas de um híbrido de Eucalyptus urophylla $\times$ E. globulus, sendo que este foi plantado em um espaçamento de 3,5 $\mathrm{m} \times 2,5 \mathrm{~m}$. Foi realizada adubação de plantio com aplicação de $300 \mathrm{~kg} \mathrm{ha}^{-1}$ de fosfato reativo e $100 \mathrm{~g} \mathrm{planta}^{-1} \mathrm{de}^{\mathrm{NPK}}$ 06:30:06, na linha de plantio. Foram realizadas duas adubações de cobertura: uma aos três meses, após implantação, com adição de $150 \mathrm{~g} \mathrm{planta}^{-1}$ de NPK 15:05:30, e outra aos 12 meses de idade das plantas, na mesma dose e com a mesma formulação da aplicação anterior.

\subsection{Metodologia de coleta da serapilheira acumulada sobre o solo (SASS)}

A determinação da serapilheira acumulada (SASS) foi realizada estacionalmente, entre março de 2007 e dezembro de 2010. As coletas foram realizadas ao final de cada estação (outonojunho; inverno-setembro; primavera-dezembro; verão-março), sendo coletadas 16 amostras, no total: quatro amostras por parcela $(20 \mathrm{~m} \times 20 \mathrm{~m})$, de forma aleatória. Para isso, utilizou-se uma moldura quadrada $(25 \mathrm{~cm} \times 25 \mathrm{~cm})$ de madeira. As amostras foram acondicionadas em sacos plásticos $\mathrm{e}$ encaminhadas ao Laboratório de Ecologia Florestal da Universidade Federal de Santa Maria, onde passaram por um processo de limpeza, visando à retirada de pedriscos, restos de solo aderido à serapilheira, raízes, etc. Após esses procedimentos, as mesmas foram colocadas em sacos de papel pardo e encaminhadas para secagem em estufa de circulação e renovação de ar, a uma temperatura de $70{ }^{\circ} \mathrm{C}$, até atingir peso constante. Foi utilizada uma balança de precisão $(0,01 \mathrm{~g})$ para determinação da massa seca das amostras.

Para a moagem e posterior análise química, foram realizadas amostras compostas com a mistura das quatro amostras de cada parcela, resultando em quatro amostras por estação. Estas foram moídas em moinho de lâminas do tipo Willey, com peneira de 30 mesh, para posterior análise dos teores de macronutrientes (N, P, K, Ca, Mg e S) e

Tabela 2. Atributos físicos do solo na área experimental localizada no município de Eldorado do Sul-RS.

Table 2. Soil Physical attributes in the experimental area located in Eldorado do Sul, RS.

\begin{tabular}{|c|c|c|c|c|c|c|c|c|c|}
\hline \multirow{2}{*}{$\begin{array}{c}\text { Profundidade } \\
(\mathrm{cm})\end{array}$} & \multicolumn{4}{|c|}{ Areia (\%) } & \multicolumn{4}{|c|}{ Silte (\%) } & \multirow{2}{*}{$\begin{array}{c}\text { Argila } \\
(\%)\end{array}$} \\
\hline & Grossa & Média & Fina & Total & Grosso & Médio & Fino & Total & \\
\hline $0-25$ & 20,4 & 14,9 & 10,9 & 46,2 & 4,6 & 4,1 & 2,9 & 11,6 & 42,2 \\
\hline $25-50$ & 12,7 & 6,1 & 4,7 & 23,5 & 4,7 & 5,1 & 4,0 & 13,8 & 62,7 \\
\hline $50-75$ & 16,1 & 9,1 & 4,6 & 29,8 & 6,8 & 7,5 & 5,1 & 19,4 & 50,8 \\
\hline $75-100$ & 28,5 & 15,7 & 5,7 & 49,9 & 12,1 & 9,2 & 4,4 & 25,7 & 24,4 \\
\hline \multirow{2}{*}{$\begin{array}{l}\text { Profundidade } \\
\qquad(\mathrm{cm})\end{array}$} & \multicolumn{2}{|c|}{$\begin{array}{l}\text { Densidade } \\
\text { aparente }\end{array}$} & \multicolumn{2}{|c|}{$\begin{array}{l}\text { Densidade } \\
\text { partículas }\end{array}$} & $\begin{array}{c}\text { Porosidade } \\
\text { total }\end{array}$ & \multicolumn{2}{|c|}{$\begin{array}{l}\text { Microporosi- } \\
\text { dade }\end{array}$} & \multicolumn{2}{|c|}{$\begin{array}{l}\text { Macroporosi- } \\
\text { dade }\end{array}$} \\
\hline & \multicolumn{4}{|c|}{$\mathrm{g} \mathrm{cm}^{-3}$} & \multicolumn{5}{|c|}{$\%$} \\
\hline $0-25$ & \multicolumn{2}{|c|}{1,41} & \multicolumn{2}{|c|}{2,74} & 48,23 & \multicolumn{2}{|c|}{18,06} & \multicolumn{2}{|c|}{30,17} \\
\hline $25-50$ & \multicolumn{2}{|c|}{1,33} & \multicolumn{2}{|c|}{2,81} & 52,62 & \multicolumn{2}{|c|}{36,16} & \multicolumn{2}{|c|}{16,45} \\
\hline $50-75$ & \multicolumn{2}{|c|}{1,37} & \multicolumn{2}{|c|}{2,84} & 51,79 & \multicolumn{2}{|c|}{36,79} & \multicolumn{2}{|c|}{15,00} \\
\hline $75-100$ & \multicolumn{2}{|c|}{1,46} & \multicolumn{2}{|c|}{2,84} & 48,54 & \multicolumn{2}{|c|}{36,91} & \multicolumn{2}{|c|}{11,63} \\
\hline
\end{tabular}


micronutrientes (B, $\mathrm{Cu}, \mathrm{Fe}, \mathrm{Mn}$ e $\mathrm{Zn}$ ). O nitrogênio foi determinado pelo método Kjeldahl (digestão sulfúrica $=\mathrm{H}_{2} \mathrm{SO}_{4}+\mathrm{H}_{2} \mathrm{O}_{2}$ ); o fósforo e o boro, por espectrofotometria ( $\mathrm{P}$ por digestão nítricaperclórica e B por digestão seca); o potássio, por fotometria de chama; o enxofre, por turbidimetria, e cálcio, magnésio, cobre, ferro, manganês e zinco, por espectrometria de absorção atômica (todos por digestão nítrica-perclórica), seguindo a metodologia descrita por Tedesco et al. (1995) e Miyazawa et al. (1999).

O cálculo do coeficiente de decomposição (K) foi realizado segundo Olson (1963), o qual considera a relação entre a serapilheira produzida anualmente, retida nos coletores, e a quantidade média de serapilheira acumulada sobre o solo. $\mathrm{O}$ modelo assume que a velocidade de decomposição da serapilheira diminui exponencialmente com o tempo, sendo que, para o desaparecimento de $50 \%$ da serapilheira acumulada, ou seja, a sua meia-vida ou vida média, o cálculo pode ser realizado através da expressão: $\mathrm{t}_{0,5}=\ln 2 / \mathrm{K}$, e o tempo de desaparecimento de $95 \%$ da serapilheira pode ser calculado por $\mathrm{t}_{0,95}=3 / \mathrm{K}$.

O cálculo do coeficiente de devolução (CD, $\mathrm{kg} \mathrm{ha} \mathrm{h}^{-1}$ ) de nutrientes via decomposição da serapilheira foi estimado pela seguinte expressão:

$\mathrm{CD}=\mathrm{A} /(\mathrm{A}+\mathrm{F})$

em que: $\mathrm{A}=$ quantidade anual de aporte de nutrientes via deposição de serapilheira $\left(\mathrm{kg} \mathrm{ha}^{-1}\right) ; \mathrm{F}=$ quantidade de nutrientes contidos na serapilheira acumulada $\left(\mathrm{kg} \mathrm{ha}^{-1}\right)$ (Chaturvedi \& Singh, 1987).

Para a determinação do tempo médio de residência (TMR, anos) do nutriente na serapilheira, foi utilizada a relação entre a quantidade de determinado nutriente na serapilheira acumulada $\left(\mathrm{kg} \mathrm{ha}{ }^{-1}\right)$ e a quantidade desse nutriente na serapilheira produzida anualmente $\left(\mathrm{kg} \mathrm{ha}{ }^{-1}\right)$, sugerida por Adams \& Attiwill (1986).

Avaliou-se, também, a quantidade de nutrientes disponibilizados (QND, $\mathrm{kg} \mathrm{ha}^{-1}$ ), via decomposição de serapilheira, durante os quatro anos de estudo:

$\mathrm{QND}=\left(\mathrm{NSPT}+\mathrm{NSA}_{0}\right)-\mathrm{NSA}_{\mathrm{f}}$

em que: NSPT $=$ Quantidade de determinado nutriente na serapilheira produzida durante todo o período de estudo, em $\mathrm{kg} \mathrm{ha}^{-1}$ (4 anos); $\mathrm{NSA}_{0}=$ Quantidade de determinado nutriente na serapilheira acumulada no início da avaliação, em kg ha-1 (ano de 2007); $\mathrm{NSA}_{\mathrm{f}}=$ Quantidade de determinado nutriente na serapilheira acumulada no final da avaliação, em kg ha-1 (ano de 2010).

A serapilheira produzida anualmente (L), a quantidade anual de aporte de nutrientes via deposição de serapilheira (A), a quantidade de determinado nutriente na serapilheira produzida anualmente (NSP) e a quantidade de determinado nutriente na serapilheira produzida durante todo o período de estudo (NSPT) podem ser verificadas no estudo realizado por Viera (2012). Para a avaliação da variação estacional da quantidade de serapilheira acumulada, dentro de cada ano de avaliação e para a média dos quatro anos, considerou-se um Delineamento Inteiramente Casualizado, em que os tratamentos corresponderam às estações do ano (verão, outono, inverno e primavera) e as repetições, às parcelas (quatro). Em razão de não haver diferença significativa das estações entre os anos, optou-se por apresentar apenas a média estacional dos quatro anos de avaliação. Utilizou-se o teste Tukey, no nível de 5\% de significância, para a comparação de médias. As análises estatísticas foram realizadas com o auxílio do programa estatístico ASSISTAT versão 7.6 beta (Silva, 2012).

\section{RESULTADOS E DISCUSSÃO}

A quantidade de serapilheira estocada sobre o solo foi, em média, de 14,0 $\mathrm{Mg} \mathrm{ha}^{-1}$ (Figura 2), estando entre os valores (4,76 a 16,7 $\mathrm{Mg} \mathrm{ha}^{-1}$ ) observados por

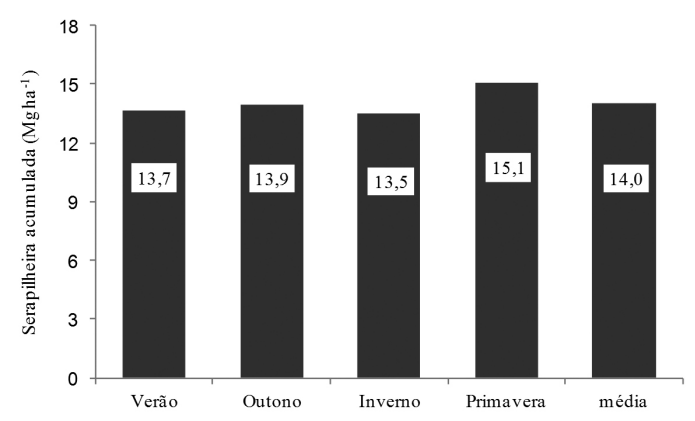

Figura 2. Variação estacional média da serapilheira acumulada em um povoamento de Eucalyptus urophylla $\times$ Eucalyptus globulus.

Figure 2. Average seasonal variation of accumulated litter in Eucalyptus urophylla $\times$ Eucalyptus globulus stand. 
outros estudos com diferentes espécies de eucalipto (Schumacher et al. (2002), Zaia \& Gama-Rodrigues (2004), Kleinpaul et al. (2005)).

A quantidade de serapilheira acumulada foi a mesma em todas as estações do ano para todos os quatro anos estudados ( $\mathrm{p}>0,05)$. Zaia \& Gama-Rodrigues (2004) também não observaram diferença no estoque de serapilheira em povoamentos de eucaliptos entre os períodos de inverno e verão. Entretanto, Witschoreck e Schumacher (2000), analisando povoamentos de Eucalyptus spp., em diferentes idades (2, 4, 6 e 8 anos), observaram um aumento acentuado no acúmulo de serapilheira, conforme o aumento da idade dos plantios (encontraram 4,05; 5,98; 11,80; $12,28 \mathrm{Mg} \mathrm{ha}{ }^{-1}$, respectivamente, para as idades citadas anteriormente).

A variação na quantidade de serapilheira acumulada entre plantios de eucalipto pode ser explicada com base na variação do clima, de sítios, de idade e de densidade de plantio, além das diferentes características genéticas de cada espécie e da estabilidade alcançada pelo povoamento (Reis \& Barros, 1990; Schumacher et al., 2002; Viera et al., 2010). Esses fatores influenciam a variação da quantidade de material depositado e o tempo necessário para a sua decomposiçãao, refletindo na maior ou menor acumulação de serapilheira sobre o solo florestal.

O somatório da quantidade de $\mathrm{Ca}$ e $\mathrm{N}$ corresponde a mais de $82 \%$ do total de macronutrientes contidos na serapilheira acumulada sobre o solo. A soma do conteúdo de $\mathrm{Mn}$ e $\mathrm{Fe}$ corresponde a aproximadamente $98 \%$ do total de micronutrientes (Tabela 3). A magnitude total do conteúdo de macronutrientes na serapilheira foi de $\mathrm{Ca}>\mathrm{N}>\mathrm{Mg}>\mathrm{K}>\mathrm{S}>\mathrm{P}$. Esta sequência é similar à mencionada por Haag (1985) em diversos estudos, havendo apenas a inversão entre as quantidades de $\mathrm{N}$ e Ca, ocasionada pelo maior conteúdo de Ca nos materiais lenhosos, os quais não foram considerados pelo autor supracitado. Dentre os micronutrientes, o ferro apresentou a maior acumulação; em parte, isto é devido à sua maior concentração em relação aos demais nutrientes. A magnitude de acúmulo de micronutrientes na serapilheira acumulada foi de $\mathrm{Fe}>\mathrm{Mn}>\mathrm{B}>\mathrm{Zn}>\mathrm{Cu}$.

A elevada quantidade de $\mathrm{Fe}$ e $\mathrm{Mn}$ presentes na serapilheira acumulada, segundo Viera et al. (2010), é decorrente das altas concentrações desses elementos no solo e/ou da contaminação das amostras com partículas de solo. Segundo os autores, a contaminação da serapilheira poderia ser causada pela aderência do solo aos resíduos vegetais, sendo de difícil separação (mesmo com a limpeza), em razão da presença de resíduos vegetais em avançado processo de decomposição.

O coeficiente de decomposição (K) médio foi de 0,54 , variando de 0,47 (durante o ano de 2009) a 0,61 (durante o ano de 2010) (Tabela 4). Para florestas tropicais, os valores de $\mathrm{K}$ geralmente são superiores a um (Pires et al., 2006) e, em povoamentos de eucaliptos, os valores são inferiores a um. Zaia \& Gama-Rodrigues (2004) encontraram valores de K

Tabela 3. Quantidade de nutrientes na serapilheira acumulada em um povoamento de Eucalyptus urophylla $\times$ Eucalyptus globulus.

Table 3. Nutrients amount in the accumulated litter in Eucalyptus urophylla $\times$ Eucalyptus globulus stand.

\begin{tabular}{|c|c|c|c|c|c|c|c|c|c|c|c|c|}
\hline \multirow{2}{*}{ Ano } & \multirow{2}{*}{$\begin{array}{l}\text { Biomassa } \\
\left(\mathrm{Mg} \mathrm{ha}^{-1}\right)\end{array}$} & \multicolumn{6}{|c|}{ Macronutrientes $\left(\mathrm{kg} \mathrm{ha}^{-1}\right)$} & \multicolumn{5}{|c|}{ Micronutrientes $\left(\mathrm{g} \mathrm{ha}^{-1}\right)$} \\
\hline & & $\mathbf{N}$ & $\mathbf{P}$ & $\mathbf{K}$ & $\mathrm{Ca}$ & Mg & $\mathbf{S}$ & B & $\mathrm{Cu}$ & $\mathrm{Fe}$ & Mn & Zn \\
\hline 2007 & 13,5 & $\begin{array}{c}103,0 \\
\pm 12,1^{1}\end{array}$ & $\begin{array}{c}4,6 \\
\pm 0,4\end{array}$ & $\begin{array}{c}19,9 \\
\pm 11,3\end{array}$ & $\begin{array}{l}139,2 \\
\pm 32,2\end{array}$ & $\begin{array}{l}21,9 \\
\pm 6,7\end{array}$ & $\begin{array}{c}9,7 \\
\pm 6,0\end{array}$ & $\begin{array}{l}222,5 \\
\pm 39,9\end{array}$ & $\begin{array}{l}102,8 \\
\pm 21,7\end{array}$ & $\begin{array}{r}12.226,9 \\
\pm 5.513,2\end{array}$ & $\begin{array}{l}6.493,8 \\
\pm 809,3\end{array}$ & $\begin{array}{l}136,5 \\
\pm 62,4\end{array}$ \\
\hline 2008 & 12,5 & $\begin{array}{l}115,8 \\
\pm 22,5\end{array}$ & $\begin{array}{c}4,5 \\
\pm 0,8\end{array}$ & $\begin{array}{l}16,6 \\
\pm 5,3\end{array}$ & $\begin{array}{c}145,3 \\
\pm 4,4\end{array}$ & $\begin{array}{l}21,8 \\
\pm 3,2\end{array}$ & $\begin{array}{c}7,9 \\
\pm 1,6\end{array}$ & $\begin{array}{c}220,0 \\
\pm 6,2\end{array}$ & $\begin{array}{l}112,6 \\
\pm 22,2\end{array}$ & $\begin{array}{l}10.301,7 \\
\pm 1.148,3\end{array}$ & $\begin{array}{l}5.707,7 \\
\pm 413,9\end{array}$ & $\begin{array}{l}188,7 \\
\pm 12,2\end{array}$ \\
\hline 2009 & 16,2 & $\begin{array}{l}123,5 \\
\pm 14,3\end{array}$ & $\begin{array}{c}5,3 \\
\pm 0,4\end{array}$ & $\begin{array}{l}16,9 \\
\pm 0,6\end{array}$ & $\begin{array}{c}169,3 \\
\pm 5,9\end{array}$ & $\begin{array}{c}30,8 \\
3,4\end{array}$ & $\begin{array}{l}8,8 \\
0,3\end{array}$ & $\begin{array}{c}235,9 \\
\pm 4,7\end{array}$ & $\begin{array}{l}136,1 \\
\pm 57,8\end{array}$ & $\begin{array}{r}15.985,3 \\
\pm 2.162,0\end{array}$ & $\begin{array}{l}7.743,9 \\
\pm 956,6\end{array}$ & $\begin{array}{l}266,7 \\
\pm 45,3\end{array}$ \\
\hline 2010 & 14,0 & $\begin{array}{l}127,3 \\
\pm 12,3\end{array}$ & $\begin{array}{c}5,9 \\
\pm 0,7\end{array}$ & $\begin{array}{l}14,0 \\
\pm 0,9\end{array}$ & $\begin{array}{r}133,5 \\
\pm 15,8\end{array}$ & $\begin{array}{l}30,9 \\
\pm 5,4\end{array}$ & $\begin{array}{c}7,5 \\
\pm 1,0\end{array}$ & $\begin{array}{l}271,7 \\
\pm 34,6\end{array}$ & $\begin{array}{l}126,9 \\
\pm 16,5\end{array}$ & $\begin{array}{r}16.445,0 \\
\pm 3.004,4\end{array}$ & $\begin{array}{l}7.739,4 \\
\pm 668,4\end{array}$ & $\begin{array}{l}251,9 \\
\pm 40,1\end{array}$ \\
\hline Média & 14,0 & 117,4 & 5,1 & 16,9 & 146,8 & 26,4 & 8,5 & 237,6 & 119,6 & $13.739,7$ & $6.921,2$ & 211,0 \\
\hline
\end{tabular}

${ }^{1}$ Desvio-padrão. 
variando entre 0,51 e 1,0, sendo que essa variação foi atribuída à diferença de concentração de lignina nos tecidos.

O tempo médio para a renovação da serapilheira $(1 / \mathrm{K})$ foi de 1,86 ano (com variação de 1,65 a 2,14 anos), estando na faixa de valores verificados na literatura. Em um povoamento de Eucalyptus grandis, com aproximadamente 20 anos de idade, em Bofete-SP, Kolm \& Poggiani (2003) encontraram um tempo médio para renovação da serapilheira de 1,77 ano. Poggiani (1985) encontrou 1,78 ano como tempo médio de renovação da serapilheira para um povoamento de Eucalyptus saligna entre 7 e 10 anos de idade.

O tempo médio para decomposição de 50 e 95\% da serapilheira foi estimado, respectivamente, em 1,29 ano (variação de 1,14 a 1,48 ano) e 5,59 anos (variação de 4,94 a 6,42 anos). Valores similares foram obtidos por Kolm \& Poggiani (2003), em plantios de Eucalyptus grandis (1,24 e 5,36 anos, para a decomposição de 50 e $95 \%$ da serapilheira, respectivamente), e por Poggiani (1985), em plantio de Eucalyptus saligna (1,2 e 5,3 anos, para a decomposição de 50 e 95\% da serapilheira, respectivamente).

O coeficiente de devolução de nutrientes é determinado pelo processo de fragmentação da serapilheira e pela liberação dos nutrientes no piso florestal (Singh et al., 2004). O potássio é o elemento com maior coeficiente de devolução $(0,52)$, seguido por $\mathrm{B}>\mathrm{Mg}>\mathrm{S}=\mathrm{Mn}>\mathrm{P}>\mathrm{N}=\mathrm{Ca}>\mathrm{Zn}>\mathrm{Cu}$ (Tabela 5); consequentemente, é o elemento que apresenta menor tempo médio de residência na serapilheira (0,91 ano) (Tabela 6).

O maior coeficiente de devolução e o menor tempo médio de residência verificados para o $\mathrm{K}$ são similares aos relatados por Zaia \& Gama-Rodrigues (2004) para o Eucalyptus grandis e o Eucalyptus pellita; entretanto, para os demais nutrientes, os valores de tempo médio de residência encontrados por esses autores são inferiores aos verificados neste estudo, o que indica que a ciclagem biogeoquímica para as espécies estudadas por esses autores é superior à deste estudo. Essa diferença verificada em relação ao estudo realizado por Zaia \& Gama-Rodrigues (2004) é ocasionada por fatores relacionados ao clima: o presente estudo foi realizado em região subtropical e,

Tabela 4. Coeficiente de decomposição (K), tempo médio de renovação (1/K) e tempo necessário para decomposição de 50 e $95 \%$ da serapilheira, em um povoamento de Eucalyptus urophylla $\times$ Eucalyptus globulus.

Table 4. Decomposition coefficient (K), mean renewal $(1 / \mathrm{K})$ and time for litter decomposition of 50 and $95 \%$ in Eucalyptus urophylla $\times$ Eucalyptus globulus stand.

\begin{tabular}{|c|c|c|c|c|c|c|}
\hline \multirow{2}{*}{ Ano } & Serapilheira & SA & \multirow{2}{*}{$\mathbf{K}$} & $1 / K$ & T0,5 & T0,05 \\
\hline & \multicolumn{2}{|c|}{$\operatorname{kg~ha}^{-1}$} & & & ano & \\
\hline 2007 & $6.870,50$ & $13.532,73$ & 0,51 & 1,97 & 1,37 & 5,91 \\
\hline 2008 & $7.226,50$ & $12.464,90$ & 0,58 & 1,72 & 1,20 & 5,17 \\
\hline 2009 & $7.576,60$ & $16.206,50$ & 0,47 & 2,14 & 1,48 & 6,42 \\
\hline 2010 & $8.500,30$ & $14.005,06$ & 0,61 & 1,65 & 1,14 & 4,94 \\
\hline Média & $7.543,50$ & $14.047,31$ & 0,54 & 1,86 & 1,29 & 5,59 \\
\hline
\end{tabular}

$\mathrm{SA}=$ Serapilheira Acumulada.

Tabela 5. Coeficiente anual de devolução de nutrientes (turnover) via decomposição da serapilheira, em um povoamento de Eucalyptus urophylla $\times$ Eucalyptus globulus.

Table 5. Nutrients turnover through litter decomposition in Eucalyptus urophylla $\times$ Eucalyptus globulus stand.

\begin{tabular}{ccccccccccc} 
Ano & $\mathbf{N}$ & $\mathbf{P}$ & $\mathbf{K}$ & $\mathbf{C a}$ & $\mathbf{M g}$ & $\mathbf{S}$ & $\mathbf{B}$ & $\mathbf{C u}$ & $\mathbf{M n}$ & $\mathbf{Z n}$ \\
\hline 2007 & 0,28 & 0,31 & 0,45 & 0,27 & 0,33 & 0,29 & 0,45 & 0,23 & 0,31 & 0,29 \\
2008 & 0,26 & 0,33 & 0,54 & 0,26 & 0,37 & 0,34 & 0,41 & 0,19 & 0,34 & 0,21 \\
2009 & 0,26 & 0,31 & 0,54 & 0,24 & 0,33 & 0,32 & 0,44 & 0,13 & 0,29 & 0,17 \\
2010 & 0,29 & 0,30 & 0,57 & 0,31 & 0,36 & 0,36 & 0,47 & 0,22 & 0,32 & 0,21 \\
Média & 0,27 & 0,31 & 0,52 & 0,27 & 0,35 & 0,32 & 0,45 & 0,19 & 0,32 & 0,21 \\
\hline
\end{tabular}


Tabela 6. Tempo médio de residência do nutriente (TMR, ano) na serapilheira, em um povoamento de Eucalyptus urophylla $\times$ Eucalyptus globulus.

Table 6. Average time of release in the litter in Eucalyptus urophylla $\times$ Eucalyptus globulus stand.

\begin{tabular}{|c|c|c|c|c|c|c|c|c|c|c|}
\hline \multirow{2}{*}{ Ano } & $\mathbf{N}$ & $\mathbf{P}$ & $\mathbf{K}$ & $\mathrm{Ca}$ & $\mathrm{Mg}$ & S & B & $\mathrm{Cu}$ & Mn & $\mathrm{Zn}$ \\
\hline & \multicolumn{10}{|c|}{ TMR (ano) } \\
\hline 2007 & 2,62 & 2,22 & 1,20 & 2,77 & 2,00 & 2,50 & 1,24 & 3,35 & 2,18 & 2,50 \\
\hline 2008 & 2,91 & 2,05 & 0,86 & 2,80 & 1,72 & 1,94 & 1,43 & 4,30 & 1,93 & 3,73 \\
\hline 2009 & 2,79 & 2,19 & 0,87 & 3,09 & 2,07 & 2,16 & 1,26 & 6,81 & 2,44 & 4,84 \\
\hline 2010 & 2,50 & 2,28 & 0,74 & 2,23 & 1,77 & 1,77 & 1,12 & 3,48 & 2,13 & 3,76 \\
\hline Média & 2,69 & 2,19 & 0,91 & 2,71 & 1,88 & 2,09 & 1,24 & 4,22 & 2,17 & 3,71 \\
\hline
\end{tabular}

Tabela 7. Quantidade de nutrientes disponibilizados (QND) via decomposição de serapilheira, durante os quatro anos de estudo, em um povoamento de Eucalyptus urophylla $\times$ Eucalyptus globulus.

Table 7. Available nutrients amount through litter decomposition, during four years of study in Eucalyptus urophylla $\times$ Eucalyptus globulus stand.

\begin{tabular}{|c|c|c|c|c|c|c|c|c|c|c|}
\hline & \multicolumn{6}{|c|}{ Macronutrientes $\left(\mathrm{kg} \mathrm{ha}^{-1}\right)$} & \multicolumn{4}{|c|}{ Micronutrientes $\left(\mathrm{g} \mathrm{ha}^{-1}\right)$} \\
\hline & $\mathbf{N}$ & $\mathbf{P}$ & $\mathbf{K}$ & $\mathrm{Ca}$ & Mg & $\mathbf{S}$ & B & $\mathrm{Cu}$ & Mn & $\mathrm{Zn}$ \\
\hline Total & 193,3 & 10,7 & 76,3 & 235,6 & 42,3 & 17,8 & 857,4 & 181,5 & $12.220,7$ & 903,9 \\
\hline Anual $^{*}$ & 48,3 & 2,7 & 19,1 & 58,9 & 10,6 & 4,4 & 214,4 & 45,4 & $3.055,2$ & 226,0 \\
\hline
\end{tabular}

${ }^{*}$ Corresponde à razão entre o total no período de estudo e o número de anos de avaliação (quatro).

dos autores citados, foi em região tropical; à espécie, pois cada espécie possui exigências nutricionais e ciclos bioquímicos distintos; à idade dos povoamentos, e às práticas silviculturais aplicadas, entre outros.

Considerando-se os quatro anos de estudos realizados, durante o período de 2007 a 2010, quando o povoamento encontrava-se com 6,5 a 9,5 anos de idade, a quantidade de nutrientes disponibilizados via serapilheira foi $235,6 \mathrm{~kg} \mathrm{ha}^{-1} \mathrm{de}$ $\mathrm{Ca}\left(58,9 \mathrm{~kg} \mathrm{ha}^{-1} \mathrm{ano}^{-1}\right)$, seguido por $\mathrm{N}, \mathrm{K}, \mathrm{Mg}, \mathrm{S}, \mathrm{Mn}$, P, Zn, B e Cu (Tabela 7).

A quantidade de nutrientes disponibilizados pela decomposição da serapilheira demonstra a importância da ciclagem biogeoquímica para o suprimento de nutrientes no sítio florestal. Segundo Zaia \& Gama-Rodrigues (2004), quanto maior a ciclagem de nutrientes, maior será a disponibilidade de nutrientes no solo para a absorção das plantas.

\section{CONCLUSÕES}

A quantidade de serapilheira acumulada sobre o solo manteve-se constante durante os quatro anos de estudo, sem apresentar influência sazonal. O estoque de Ca e $\mathrm{N}$ corresponde a mais de $82 \%$ do total de macronutrientes, e de Mn e Fe corresponde a aproximadamente $98 \%$ do total de micronutrientes, contidos na serapilheira acumulada. A serapilheira apresentou baixo coeficiente de decomposição $(\mathrm{K}=0,54)$, característico das diferentes espécies de eucalipto. O potássio apresentou o maior coeficiente de devolução e o menor tempo médio de residência na serapilheira.

\section{STATUS DA SUBMISSÃO}

Recebido: $10 / 12 / 2012$

Aceito em: 26/06/2013

Publicado: 30/09/2013

\section{AUTOR(ES) PARA CORRESPONDÊNCIA}

\section{Márcio Viera}

Departamento Multidisciplinar, Universidade Federal de Santa Maria - UFSM, Rua Francisco Guerino, 407, Centro, CEP 97195-000, Silveira Martins, RS, Brasil e-mail: marcio.viera@ufsm.br 


\section{REFERENNCIAS}

Adams MA, Attiwill PM. Nutrient cycling and nitrogen mineralization in eucalypt forests of south-eastern Australia: I. Nutrient cycling and nitrogen turnover. Plant and Soil 1986; 92(3): 319-339. http://dx.doi. org/10.1007/BF02372482

Campos ML, Marchi G, Lima DM, Silva CA. Ciclagem de nutrientes em floretas e pastagens. Lavras: Universidade Federal de Lavras; 2004. (Boletim Agropecuário, n. 64).

Chaturvedi OP, Singh JS. Structure and function of pine forest of central Himalaya. II. Nutrient dynamics. Annals of Botany 1987; 60(3): 253-267.

Dutta RK, Agrawal M. Litterfall, litter decomposition and nutrient release in five exotic plant species planted on coal mine spoils. Pedobiologia 2001; 45: 298-312. http://dx.doi.org/10.1078/0031-4056-00088

Fundação Estadual de Pesquisa Agropecuária do Rio Grande do Sul - FEPAGRO. Atlas climático: Rio Grande do Sul. Fepagro: Porto Alegre; 2012.

Figueiredo Filho A, Morais GF, Schaaf LB, Figueiredo DJ. Avaliação Estacional da Deposição de Serapilheira em uma Floresta Ombrófila Mista Localizada no Sul do Paraná. Ciência Florestal 2003; 13(1): 11-18.

Guo LB, Sims REH. Eucalypt litter decomposition and nutrient release under a short rotation forest regime and effluent irrigation treatments in New Zealand: II. internal effects. Soil Biology and Biochemistry 2002; 34: 913-922. http://dx.doi.org/10.1016/S0038-0717(02)00023-8

Haag HP. Ciclagem de nutrientes em florestas tropicais. Campinas: Fundação Cargill; 1985.

Kleinpaul IS, Schumacher MV, Brun EJ, Brun FGK, Kleinpaul JJ. Suficiência amostral para coletas de serapilheira acumulada sobre o solo em Pinus elliotti Engelm, Eucalyptus sp. e Floresta Estacional Decidual. Revista Árvore 2005; 29(6): 965-972. http://dx.doi. org/10.1590/S0100-67622005000600016

Koehler CW, Reissmann CB, Koehler HS. Deposição de resíduos orgânicos (serapilheira) e nutrientes em plantio de Araucaria angustifolia em função do sítio. Revista do Setor de Ciências Agrárias 1987; 9(1): 89-94.

Kolm L, Poggiani F. Ciclagem de nutrientes em povoamentos de Eucalyptus grandis submetidos à prática de desbastes progressivos. Scientia Forestalis 2003; 63: 79-93.

Mason CF. Decomposição. Tradução de Otávio Antonio de Camargo. São Paulo; 1980. (Temas de biologia, v. 18).

Miyazawa M, Pavan MA, Muraoka T, Carmo CAFS, Mello NJ. Análises químicas de tecido vegetal. In: Chitolina JC, Silva FC, Prata F, Muraoka T, Vitti GC, organizadores. Manual de análises químicas de solos, plantas e fertilizantes. Brasília: Embrapa Comunicação para Transferência de Tecnologia; 1999.

Moreno JA. Clima do Rio Grande do Sul. Porto Alegre: Secretaria da Agricultura; 1961.

Neves EJM, Martins EG, Reissmann CB. Deposição de serapilheira e de nutrientes de duas espécies da Amazônia. Boletim de Pesquisa Florestal 2001; 43: 47-60.

Olson JS. Energy storage and the balance of producers and decomposers in ecological systems. Ecology 1963; 44(2): 322-330. http://dx.doi. org/10.2307/1932179

O'Connell AM. Nutrient dynamics in decomposing litter in Karri (Eucalyptus diversicolor F. Muell.) forests of South-westren Australia. Journal of Ecology 1988; 76: 1186-1203. http://dx.doi. org/10.2307/2260642

Pires LA, Britez RM, Martel G, Pagano SN. Produção, acúmulo e decomposição da serapilheira em uma restinga da Ilha do Mel, Paranaguá, PR, Brasil. Acta Botânica Brasilica 2006; 20(2): 173-184. http://dx.doi. org/10.1590/S0102-33062006000100016

Poggiani F. Ciclagem de nutrientes em ecossistemas de plantações florestais de Eucalyptus e Pinus: Implicações silviculturais [tese]. São Paulo: Universidade de São Paulo; 1985.

Pritchett WL. Properties and management of forest soils. New York: John Wiley; 1979.

Reis MGF, Barros NF. Ciclagem de nutrientes em plantios de eucalipto. In: Barros NF, Novais RF, editores. Relação Solo-eucalipto. Viçosa: Folha de Viçosa; 1990.

Schumacher MV, Bauermann GC, Copetti L, Brun EJ, König FG. Fracionamento da serapilheira em três espécies de eucalipto no município de Santa Maria-RS: Eucalyptus urophylla, Eucalyptus cloesiana e Eucalyptus grandis. In: Ciclo de atualização florestal do CONESUL. Santa Maria; 2002. p. 173-180.

Silva FAS. ASSISTAT versão 7.6 beta. Campina Grande: DEAG - CTRN - Universidade Federal de Campina Grande, Campus de Campina Grande; 2012.

Singh RK, Dutta RK, Agrawal M. Litter decomposition and nutrient release in relation to atmospheric deposition of $\mathrm{S}$ and $\mathrm{N}$ in a dry tropical region. Pedobiologia 2004; 48(4): 305-311. http://dx.doi. org/10.1016/j.pedobi.2004.03.003

Sociedade Brasileira de Ciência do Solo - SBCS. Comissão de química e fertilidade do solo. Manual de adubação e calagem para os estados do Rio Grande do Sul e de Santa Catarina. 10. ed. Porto Alegre; 2004.

Streck EV, Kämpf N, Dalmolin RSD, Kklant E, Nascimento PC, Scheneider P, et al. Solos do Rio Grande do Sul. 2. ed. Porto Alegre: EMATER/RS; 2008. 
Tedesco MJ. Gianello C, Bissani CA, Bohnen H, Volkweiss SJ. Análise de solo, plantas e outros materiais. Porto Alegre: Departamento de Solos, UFRGS; 1995. (Boletim Técnico).

Viera M. Dinâmica nutricional em um povoamento híbrido de Eucalyptus urophylla $x$ Eucalyptus globulus em Eldorado do Sul-RS, Brasil [tese]. Santa Maria: Universidade Federal de Santa Maria; 2012.

Viera M, Caldato SL, Rosa SF, Kanieski MR, Araldi DB, Santos SR, et al. Nutrientes na serapilheira em um fragmento de floresta estacional decidual, Itaara, RS. Ciência Florestal 2010; 20(4): 611-619.

Viera M, Schumacher MV. Concentração e retranslocação de nutrientes em acículas de Pinus taeda L.. Ciência Florestal 2009: 19(4): 375-382.
Viera M, Schumacher MV. Deposição de serapilheira e de macronutrientes em um povoamento de acácianegra (Acacia mearnsii De Wild.) no Rio Grande do Sul. Ciência Florestal 2010; 20(2): 225-233.

Witschoreck R, Schumacher MV. Estimativa do carbono da serapilheira em florestas de eucalipto de diferentes idades. In: Anais do $8^{\circ}$ Congresso Florestal Estadual de Nova Prata; 2000; Nova Prata. Nova Prata; 2000.

Zaia FC, Gama-Rodrigues AC. Ciclagem e balanço de nutrientes em povoamentos de eucalipto na região norte fluminense. Revista Brasileira Ciência Solo 2004; 28(5): 843-852. http://dx.doi.org/10.1590/ S0100-06832004000500007

Vitousek PM, Sanford RL. Nutrient cycling in moist tropical forest. Annual Review Systems 1986; 17: 137-167 http://dx.doi.org/10.1146/annurev.es.17.110186.001033 